\title{
Gestión Del Conocimiento En La Administración Pública: Una Revisión Sistemática
}

Knowledge management in public administration: a systematic review

\section{Edith Delia Chavez Ramirez}

Maestra en Gestión Pública, Universidad César Vallejo, Lima, Perú edithdeli@gmail.com, ORCID: https://orcid.org/0000-0002-3483-0825

https://scholar.google.es/citations?hl=es\&authuser=1\&user=qnSQmTgAAA AJ

\section{Lucila Rojas Delgado}

Maestra en Gestión de Servicios de Salud, Universidad Cesar Vallejo, Lima, Perú.

ORCID: 0000-0002-4575-3722

https://scholar.google.es/citations?view_op=list_works\&hl=es\&user=dcBAI u8AAAAJ

\section{Antiona Valderrama, Daniela Milagros}

Maestra en administración de la educación, Universidad Cesar Vallejo, Lima, Perú.

ORCID: 0000- 0002- 1189- 4789

https://scholar.google.es/citations?user=JL0muMIAAAAJ\&hl=es\&oi=ao

\section{Chavez Ramirez, Marlene}

Licenciada en Contabilidad, Universidad Cesar Vallejo, Lima, Perú.

ORCID: 0000-0001-9632-6272

https://scholar.google.es/citations?hl=es\&user=KdicByQAAAAJ

\section{Revista Iberoamericana de la Educación \\ Vol - Especial 12021 \\ e-ISSN: 2737-632x}

\begin{abstract}
Knowledge Management is a tool widely used by public and private institutions, optimizing their results and generating added value in the organization thanks to the experiences and skills of the collaborators, which motivated the investigation by the cited authors about the management of the knowledge in public administration. A systematic review was carried out in databases on knowledge management in public administration from the
\end{abstract}


period 2011 to 2021. The research is based on the methodology of Kitchenham (2004) and Okoli and Schabram (2010). The necessary results with the empirical and theoretical information in public administration policies where knowledge management is used. The results show that knowledge management contributes significantly to the efficiency of public administration institutions.

Key words: Knowledge management, public administration, public sector, public institutions.

Resumen: La Gestión del conocimiento es una herramienta muy utilizada por las instituciones públicas y privadas, optimizando sus resultados y generando valor agregado en la organización gracias a las experiencias y habilidades de los colaboradores, lo que motivó la indagación por parte de autores citados sobre la gestión del conocimiento en la administración pública. Se realizó la revisión sistemática en bases de datos sobre la gestión del conocimiento en la administración pública del periodo 2011 al 2021. La investigación se fundamente en la metodología de Kitchenham (2004) y Okoli y Schabram (2010). Los resultados contribuyen con la información empírica y teórica en políticas de la administración pública donde se usa la gestión del conocimiento. Los resultados muestran que la gestión del conocimiento contribuye significativamente en la eficiencia de las instituciones de la administración pública.

Palabras clave: Gestión del conocimiento, administración pública, sector público, instituciones públicas.

\section{INTRODUCTION}

En los últimos años, en el ámbito de la llamada economía del conocimiento, la gestión del conocimiento se ha convertido en uno de los más importantes 
temas a investigar y en el paradigma de gestión por excelencia que está presente en las organizaciones y la gestión de las instituciones (Chávez Ramírez, 2018).

La gestión del conocimiento se ha convertido en un recurso importante de las instituciones públicas, que proveen una ventaja competitiva sostenible que contribuye al desarrollo de las instituciones de la administración pública (Rubier Valdés, 2019). Y sobre la base de indicadores presentados en la revisión sistemática es resultado de la indagación bibliográfica realizado en diversas fuentes especializadas, textos científicos y revisas indexadas, que facilitan la caracterización y concreción epistémica del objeto de la investigación.

Como resultado de la revisión sistemática muestra la necesidad de las instituciones de la administración pública implementar adecuadamente la gestión del conocimiento, el cual no solo se da en el sector privado, sino también se ha utilizado ampliamente en la gestión pública a través de organizaciones públicas o gubernamentales, como educación, salud, infraestructura, economía, entre otros, que han aprovechado las ventajas de la gestión del conocimiento (Siregar et al., 2019), por ello, la gestión del conocimiento constituye una estrategia de innovación que incluye acciones que permiten compartir información, algunas experiencias y conocimiento individuales, por lo que es importante valorar el capital intelectual que permita generar valor agregado a la institución. (Zuluaga López, 2019).

El objetivo de la gestión del conocimiento no es necesariamente para gestionar todo el conocimiento, sino el conocimiento que es más importante para la organización (Mustafa et al., 2015). La identificación de las variables que se presenta desde la tesis doctoral que estudia la gestión del conocimiento, 
las TIC y la productividad laboral, donde se pretende determinar la incidencia de la gestión del conocimiento y las Tecnologías de la Información en la productividad laboral.

De esta forma, el objetivo del presente artículo según la revisión sistemática busca determinar cómo influye la gestión del conocimiento en la administración pública, donde existe una constante interacción de los datos y la información que son diferentes del conocimiento, pero se encuentra interrelacionados (Hegazy \& Ghorab, 2014).

\section{VARIABLES DE ESTUDIO}

Al analizar los artículos seleccionados se presentan puntos en común como el planteamiento de problemas donde los investigadores intentan describir como la gestión del conocimiento contribuye positivamente en la administración pública. A continuación, se muestra la tabla de variables de los estudios objeto de comparación en la que se presenta como la gestión del conocimiento se convierte en un potencial elemento para el éxito de las instituciones de la administración pública.

Tabla $\mathbf{N}^{\circ}$ 1. Variables

\begin{tabular}{|l|l|l|l|l|}
\hline Tema & Autor & Año & País & Variables \\
\hline $\begin{array}{l}\text { Gestión del } \\
\text { conocimiento: }\end{array}$ & $\begin{array}{l}\text { María } \\
\text { Cisneros, Luz } \\
\text { una apuesta desde } \\
\text { la gemberthy, } \\
\text { para la educación }\end{array}$ & $\begin{array}{l}\text { Pargye } \\
\text { Chaguendo }\end{array}$ & Colombia & $\begin{array}{l}\text { Gobernanza, gestión } \\
\text { del conocimiento, } \\
\text { apropiación social. }\end{array}$ \\
\hline
\end{tabular}




\begin{tabular}{|c|c|c|c|c|}
\hline $\begin{array}{l}\text { en salud en el } \\
\text { Cauca }\end{array}$ & & & & \\
\hline $\begin{array}{l}\text { Digital } \\
\text { Transformation } \\
\text { and Knowledge } \\
\text { Management } \\
\text { in the Public } \\
\text { Sector }\end{array}$ & $\begin{array}{l}\text { Anna } \\
\text { Alvarenga, } \\
\text { Florinda } \\
\text { Matos,Radu } \\
\text { Godina, Joao } \\
\text { C.O, Matias }\end{array}$ & 2020 & Portugal & $\begin{array}{l}\text { Transformación } \\
\text { digital, conocimiento } \\
\text { administrativo, } \\
\text { gobierno digital, } \\
\text { sector público. }\end{array}$ \\
\hline $\begin{array}{l}\text { The effectiveness } \\
\text { of knowledge } \\
\text { management in } \\
\text { the public sector }\end{array}$ & $\begin{array}{l}\text { Paul Joseph } \\
\text { Mc Evoy, } \\
\text { Mohamed } \\
\text { A.F, Ragab \& } \\
\text { Amr Arisha }\end{array}$ & 2018 & Irlanda & $\begin{array}{l}\text { Conocimiento, } \\
\text { gestión, sector } \\
\text { público, intervención } \\
\text { gubernamental. }\end{array}$ \\
\hline $\begin{array}{l}\text { La gestión del } \\
\text { conocimiento, una } \\
\text { política } \\
\text { organizacional } \\
\text { para la empresa de } \\
\text { hoy }\end{array}$ & $\begin{array}{l}\text { Erica } \\
\text { Agudelo, } \\
\text { Alejandro } \\
\text { Valencia }\end{array}$ & 2018 & Chile & $\begin{array}{l}\text { Gestión del } \\
\text { conocimiento, } \\
\text { modelo gerencial, } \\
\text { sociedad } \\
\text { conocimiento, } \\
\text { organización. }\end{array}$ \\
\hline $\begin{array}{l}\text { La gestión del } \\
\text { conocimiento en } \\
\text { las organizaciones } \\
\text { y las regiones }\end{array}$ & $\begin{array}{l}\text { Omaira, } \\
\text { Calvo }\end{array}$ & 2018 & Colombia & $\begin{array}{ll}\text { Gestión } & \text { del } \\
\text { conocimiento, } & \\
\text { regiones, } & \\
\text { organizaciones } & \text { del } \\
\text { conocimiento. } & \end{array}$ \\
\hline $\begin{array}{ll}\text { Incidencia de } & \text { las } \\
\text { prácticas } & \text { de } \\
\text { Gestión } & \text { del }\end{array}$ & $\begin{array}{l}\text { Andrea } \\
\text { Echevarri, } \\
\text { Nelson }\end{array}$ & 2018 & Colombia & $\begin{array}{l}\text { Conocimiento } \\
\text { organizacional, } \\
\text { gestión }\end{array}$ \\
\hline
\end{tabular}




\begin{tabular}{|c|c|c|c|c|}
\hline $\begin{array}{l}\text { conocimiento } \\
\text { sobre la } \\
\text { creatividad } \\
\text { organizacional }\end{array}$ & $\begin{array}{l}\text { Lozada, José } \\
\text { Arias }\end{array}$ & & & $\begin{array}{l}\text { conocimiento, } \\
\text { aprendizaje continuo, } \\
\text { gestión } \\
\text { competencias. }\end{array}$ \\
\hline $\begin{array}{l}\text { Gestión del } \\
\text { conocimiento en } \\
\text { la administración } \\
\text { pública de los } \\
\text { municipios de la } \\
\text { Región de la Gran } \\
\text { Santa rosa/RS }\end{array}$ & $\begin{array}{l}\text { Neddisson } \\
\text { Gessi, Mauro } \\
\text { Nuske, } \\
\text { Nelson } \\
\text { Thesing, } \\
\text { Sergio } \\
\text { Allebrandt, } \\
\text { Daniel } \\
\text { Baggio }\end{array}$ & 2017 & Brasil & $\begin{array}{l}\text { Conocimiento, } \\
\text { gestión, } \\
\text { ayuntamiento. }\end{array}$ \\
\hline $\begin{array}{l}\text { Knowledge } \\
\text { Management In } \\
\text { Public } \\
\text { Administration } \\
\text { Institutions }\end{array}$ & $\begin{array}{l}\text { Juroslava, } \\
\text { Buckova }\end{array}$ & 2015 & Eslovaquia & $\begin{array}{l}\text { Conocimiento, } \\
\text { estructura } \\
\text { organizacional, } \\
\text { administración } \\
\text { pública } \\
\text { infraestructura } \\
\text { tecnológica }\end{array}$ \\
\hline $\begin{array}{l}\text { Gestión del } \\
\text { conocimiento en } \\
\text { el tercer sector de } \\
\text { la competitividad } \\
\text { a la eficiencia } \\
\text { organizacional }\end{array}$ & $\begin{array}{l}\text { Cristian } \\
\text { Bedoya }\end{array}$ & 2015 & Colombia & $\begin{array}{l}\text { Gestión del } \\
\text { conocimiento, } \\
\text { conocimiento, } \\
\text { eficiencia, eficacia. }\end{array}$ \\
\hline $\begin{array}{lr}\text { Gestión } & \text { del } \\
\text { conocimiento } & y\end{array}$ & $\begin{array}{ll}\text { Ero } & \text { Del } \\
\text { Castro, } & \text { Alí }\end{array}$ & & & $\begin{array}{ll}\text { Gestión } & \text { del } \\
\text { conocimiento, } & \end{array}$ \\
\hline
\end{tabular}




\begin{tabular}{|c|c|c|c|c|}
\hline $\begin{array}{l}\text { uso de Tic's en la } \\
\text { empresa privada y } \\
\text { administración } \\
\text { pública }\end{array}$ & $\begin{array}{l}\text { García, Jaime } \\
\text { Martinez, } \\
\text { Douglas } \\
\text { Uzcátegui }\end{array}$ & 2014 & Venezuela & $\begin{array}{ll}\text { tecnologías de } & \text { la } \\
\text { información } & \text { y } \\
\text { comunicación } & \text { y } \\
\text { administración } & \\
\text { pública. } & \end{array}$ \\
\hline
\end{tabular}

De acuerdo al análisis de las variables que se presentan en la tabla anterior, destaca Cisneros-Cisneros et al., (2020), quien hace referencia a los conocimientos institucionales, saberes, sabiduría innata de las comunidades para la construcción de una sociedad más equitativa y por ende una mejor gobernanza. En otra posición de un estudio investigativo Alvarenga et al. (2020), indica que la transformación digital tiene un impacto positivo en la gestión del conocimiento de funcionarios de la gestión pública, lo que contribuye al fortalecimiento de las empresas e instituciones. Este autor Mc Evoy et al., (2018), analiza la eficacia de la gestión del conocimiento a través de una nueva taxonomía, realiza una revisión detallada de la literatura sobre la gestión del conocimiento en el sector público, asimismo analiza como la gestión del conocimiento se dinamiza en el sector salud, educación y gobierno, destacando el efecto de la gestión del conocimiento en el sector público.

Se sistematizaron además investigaciones vinculadas a la gestión del conocimiento en las organizaciones y regiones Ceballos \& Arias, (2018) y Calvo Giraldo, (2018), en ambas investigaciones se estudia la gestión del conocimiento como eje central y su desarrollo a nivel empresarial y regional, los autores consideran que la gestión del conocimiento se convierte en una estrategia del modelo gerencial que no solo contribuye al direccionamiento 
de la empresa sino también al entendimiento de la realidad donde se desarrolla la empresa.

Por su parte, Echeverri et al., (2018) presenta como variable la gestión del conocimiento como parte del conocimiento organizacional, lo que contribuye a la generación de ideas nuevas y útiles. Gessi et al.,(2017) analiza como la gestión del conocimiento se está gestionando en el gobierno municipal, lo que permite mejorar el desempeño y mejorar la estructura organizativa. Mientras que Bučková, (2015) analiza la variable de la gestión del conocimiento como una necesidad de ser introducida en la administración pública, lo que contribuye a dar respuesta a la demandas de los ciudadanos sobre la cantidad y calidad de la información.

Se consideró la publicación de Bedoya-Dorado, (2015) que identifica los aportes de la gestión del conocimiento a la eficiencia en organizaciones del sector público. De la misma forma Ero Del Castro, et al, (2014) analiza la variable de la gestión del conocimiento como un factor de éxito de las organizaciones o instituciones, lo que significa gestionar adecuadamente el flujo de información ayudando a la instituciones a reaccionar adecuadamente a los tiempos cambiantes.

\section{METODOLOGÍA}

En la presente investigación documental, se realizó una búsqueda sistematizada de literatura científica siguiendo un esquema metodológico y una escrupulosa selección de artículos científicos, en revistas indexadas a bases de datos como SciELO, Scopus y Redalyc, donde solo se seleccionaron las revistas que se acercaban con las variables de interés descartando el resto de revistas que no tenían las variables de estudio. 
Para la revisión en las bases de datos se empleó el descriptor "knowledge management" en el título del artículo. Asimismo, se cruzó información con del descriptor "public administration" los resultados de la búsqueda pertenecen a revistas de acceso abierto, tanto en idioma inglés y castellano, asimismo, se consideró los artículos científicos publicados en entre el 2010 al 2020.

A continuación, se presenta la tabla de los indicadores que se consideraron más importes de los estudios objeto de comparación (se muestran los estudios en relación al orden de autores referenciados en la tabla $\mathrm{N}^{\circ} 1$ ):

Tabla $\mathbf{N}^{\circ}$ 2. Variables

\begin{tabular}{|c|c|c|c|}
\hline Tema & $\begin{array}{c}\text { Tipo de } \\
\text { investigación }\end{array}$ & $\begin{array}{l}\text { Unidades de } \\
\text { observación }\end{array}$ & $\begin{array}{c}\text { Métodos e } \\
\text { instrumentos }\end{array}$ \\
\hline $\begin{array}{c}\text { Gestión del } \\
\text { conocimiento: una } \\
\text { apuesta desde la } \\
\text { gobernanza para la } \\
\text { educación en salud } \\
\text { en el Cauca }\end{array}$ & Cualitativo & $\begin{array}{c}\text { Gobernanza, } \\
\text { comunidades } \\
\text { caucanas } \\
\text { campesinas }\end{array}$ & $\begin{array}{c}\text { Revisión } \\
\text { documental } \\
\text { Teórico }\end{array}$ \\
\hline $\begin{array}{c}\text { Digital } \\
\text { Transformation } \\
\text { and Knowledge } \\
\text { Management } \\
\text { in the Public } \\
\text { Sector }\end{array}$ & $\begin{array}{c}\text { Cuantitativo, } \\
\text { descriptivo }\end{array}$ & $\begin{array}{c}\text { Instituciones de } \\
\text { administración } \\
\text { públicas }\end{array}$ & $\begin{array}{c}\text { Revisión } \\
\text { bibliográfica y } \\
\text { documental }\end{array}$ \\
\hline $\begin{array}{c}\text { The effectiveness } \\
\text { of knowledge } \\
\text { management in the } \\
\text { public sector }\end{array}$ & Cualitativo & $\begin{array}{l}\text { Organizaciones } \\
\text { públicas }\end{array}$ & $\begin{array}{c}\text { Revisión } \\
\text { bibliográfica y } \\
\text { documental }\end{array}$ \\
\hline $\begin{array}{c}\text { La gestión del } \\
\text { conocimiento, una }\end{array}$ & Estudio de casos & $\begin{array}{c}\text { Instituciones } \\
\text { privadas }\end{array}$ & $\begin{array}{l}\text { Grupos focales, } \\
\text { observación, }\end{array}$ \\
\hline
\end{tabular}




\begin{tabular}{|c|c|c|c|}
\hline $\begin{array}{c}\text { política } \\
\text { organizacional } \\
\text { para la empresa de } \\
\text { hoy }\end{array}$ & & & $\begin{array}{l}\text { entrevista, } \\
\text { encuestas }\end{array}$ \\
\hline $\begin{array}{c}\text { La gestión del } \\
\text { conocimiento en } \\
\text { las organizaciones } \\
\text { y las regiones }\end{array}$ & Cualitativo & $\begin{array}{c}\text { Organizaciones, } \\
\text { instituciones } \\
\text { regionales }\end{array}$ & $\begin{array}{c}\text { Revisión } \\
\text { bibliográfica y } \\
\text { documental }\end{array}$ \\
\hline $\begin{array}{l}\text { Incidencia de las } \\
\text { prácticas de } \\
\text { Gestión del } \\
\text { conocimiento } \\
\text { sobre la } \\
\text { creatividad } \\
\text { organizacional }\end{array}$ & Cuantitativo & $\begin{array}{c}\text { Empresas a nivel } \\
\text { nacional }\end{array}$ & $\begin{array}{c}\text { Ecuaciones } \\
\text { estructurarles, } \\
\text { mínimos cuadrado }\end{array}$ \\
\hline $\begin{array}{c}\text { Gestión del } \\
\text { conocimiento en la } \\
\text { administración } \\
\text { pública de los } \\
\text { municipios de la } \\
\text { Región de la Gran } \\
\text { Santa rosa/ RS }\end{array}$ & $\begin{array}{c}\text { Cuantitativa y } \\
\text { estudios de caso }\end{array}$ & $\begin{array}{l}\text { Municipios de la } \\
\text { región y } \\
\text { funcionarios } \\
\text { públicos. }\end{array}$ & $\begin{array}{c}\text { Revisión } \\
\text { bibliográfica y } \\
\text { documental }\end{array}$ \\
\hline $\begin{array}{c}\text { Knowledge } \\
\text { Management In } \\
\text { Public } \\
\text { Administration } \\
\text { Institutions }\end{array}$ & Cuantitavo & $\begin{array}{l}\text { Empresas e } \\
\text { instituciones y } \\
\text { profesionales del } \\
\text { sector público. }\end{array}$ & Teórico, encuestas \\
\hline $\begin{array}{c}\text { Gestión del } \\
\text { conocimiento en el } \\
\text { tercer sector de la } \\
\text { competitividad a la } \\
\text { eficiencia } \\
\text { organizacional }\end{array}$ & $\begin{array}{l}\text { Cualitativo, } \\
\text { exploratorio y } \\
\text { descriptivo }\end{array}$ & $\begin{array}{l}\text { Organizaciones del } \\
\text { tercer sector }\end{array}$ & $\begin{array}{l}\text { Análisis de } \\
\text { contenido, } \\
\text { revisión } \\
\text { documental }\end{array}$ \\
\hline
\end{tabular}




\begin{tabular}{|c|c|c|c|}
\hline $\begin{array}{c}\text { Gestión del } \\
\text { conocimiento y }\end{array}$ & Descriptivo & $\begin{array}{c}\text { Instituciones } \\
\text { públicas y privadas }\end{array}$ & $\begin{array}{c}\text { Revisión } \\
\text { bibliográfica y } \\
\text { documental }\end{array}$ \\
$\begin{array}{c}\text { uso de Tic's en la } \\
\text { empresa privada y } \\
\text { administración } \\
\text { pública }\end{array}$ & & & \\
\hline
\end{tabular}

Los artículos que se han revisado contienen diversas metodologías, destacan las metodologías relacionadas a estudios empíricos, revisión bibliográfica y documental, que son documentos de gran importancia que contribuye al desarrollo del objetivo de la investigación. En ese sentido, Cisneros-Cisneros et al., (2020), presenta un estudio cualitativo, basado en un profundo análisis teórico-práctico debido al vacío pragmático que identificó, donde los componentes de la gestión del conocimiento permiten llenar el vacío conceptual que es aplicado a la gobernanza de las comunidades campesinas de Cauca.

Por su parte, Alvarenga et al. (2020), Mc Evoy et al., (2019), Calvo Giraldo, (2018), Gessi et al.(2017) y Ero Del Castro, et al, (2014), realizaron un estudio cualitativo, donde usaron una metodología basada en la revisión bibliográfica y documental, siendo los datos extraidos a través de entrevistas, grupos focales de las instituciones de la gestión pública y privada de ámbito nacional y regional. Mientras que, Calvo Giraldo, (2018), realizaron un estudio de investigación enfocado en el estudio de casos, para la obtención de los datos, recurrieron a la observación, la técnica de la encuesta y entrevistas a través de grupos focales. Echeverri et al., (2018), a través de su estudio cualitativo, aplicó la técnica de ecuaciones estructurales a través del método de mínimos cuadrados para determinar la incidencia de las prácticas de la gestión del conocimiento sobre la creatividad organizacional. Destaca el trabajo de Bučková, (2015) un estudio cuantitativo, con aporte teórico con alto nivel de 
análisis donde se hace uso de encuestas para la obtención de resultados. Ero Del Castro, et al, (2014), en su investigación realiza una revisión documental en fuentes bibliográficas y electrónicas, lo que le permite hacer una reflexión de la gestión del conocimiento a través del uso de las TIC's.

La aplicación de diversas metodologías utilizadas en los artículos seleccionados es intencional y centrada en reconocer las diversas formas de recoger información auténtica, tomando en cuenta las revisiones bibliográficas lo que permite tener un número grande de fuentes de información y recursos para el planteamiento de la investigación de lo cual Cisneros-Cisneros et al., (2020), Alvarenga et al. (2020), Mc Evoy et al., (2018), Calvo Giraldo, (2018), Gessi et al.,(2017) y Ero Del Castro, et al, (2014), aportaron significativamente con sus estudios profundos sobre las revisiones bibliográficas, por otro lado, Ceballos \& Arias, (2018), Echeverri et al., (2018), Bučková, (2015) y Bedoya-Dorado, (2015), presentaron investigaciones de tipo cuantitativo, estudios de casos con una exhaustiva revisión documental, estudios empíricos con aplicación de recojo de información a través de entrevistas, grupos focales y fichas de observación.

Los estudios permiten clasificar los diferentes tipos, métodos e instrumentos que los investigadores utilizan para la recolección de datos en base a una variable, donde se pone en evidencia una diversidad de investigaciones que se desarrollan sobre la problemática de la presente investigación.

Resultados De los artículos seleccionados, los autores muestran los resultados y los socializan de forma precisa y clara ante la comunidad científica. Por ello, se realizó una tabla de los resultados más importantes:

Tabla $\mathbf{N}^{\circ}$ 3. Tabla de resultados 


\begin{tabular}{|c|c|}
\hline Tema & Resultados \\
\hline $\begin{array}{l}\text { Gestión del conocimiento: } \\
\text { una apuesta desde la } \\
\text { gobernanza para la } \\
\text { educación en salud en el } \\
\text { Cauca }\end{array}$ & $\begin{array}{l}\text { El conocimiento en salud se ha convertido en una } \\
\text { importante herramienta de aprendizaje, lo que es } \\
\text { considerado como una herramienta del aprendizaje y } \\
\text { recurso indispensable para la toma de decisiones que } \\
\text { aporta valor dentro de las entidades públicas. Entre } \\
\text { los resultado del estudio se evidenció que el } \\
\text { conocimiento adquirido en salud se ha asimilado a } \\
\text { partir de la influencia directa de la oralidad, el } \\
\text { lenguaje y la etnoculturalidad. }\end{array}$ \\
\hline $\begin{array}{l}\text { Digital Transformation and } \\
\text { Knowledge Management } \\
\text { in the Public Sector }\end{array}$ & $\begin{array}{l}\text { Los resultados muestran que la investigación sobre el } \\
\text { tema aún se encuentra en una etapa exploratoria } \\
\text { debido a la falta de estudios que relacionen el } \\
\text { gobierno digital con la efectividad de la gestión del } \\
\text { conocimiento en el sector público, los resultados } \\
\text { también muestran que el éxito del gobierno digital } \\
\text { parece estar relacionado con la calidad de la gestión } \\
\text { del conocimiento de las organizaciones, que se } \\
\text { complementan entre sí para importantes mejoras en el } \\
\text { sector público. }\end{array}$ \\
\hline $\begin{array}{l}\text { The effectiveness of } \\
\text { knowledge management in } \\
\text { the public sector }\end{array}$ & $\begin{array}{l}\text { Se ha logrado determinar que existe dificultad para } \\
\text { medir la productividad por parte del sector público, la } \\
\text { cultura del sector público está vinculada a su } \\
\text { naturaleza jerárquica estructurada, procesos formales } \\
\text { de toma de decisiones y tendencia burocrática. }\end{array}$ \\
\hline $\begin{array}{l}\text { La gestión del conocimiento, } \\
\text { una política organizacional } \\
\text { para la empresa de hoy }\end{array}$ & $\begin{array}{l}\text { El proceso de compartir información es una actividad } \\
\text { establecida como una generación de conocimiento, de } \\
\text { los resultados los encuestados en su mayoría perciben } \\
\text { que la organización aprovecha el conocimiento que }\end{array}$ \\
\hline
\end{tabular}




\begin{tabular}{|c|c|}
\hline & $\begin{array}{l}\text { tienen las personas y que la organización aprende de } \\
\text { la interacción con el entorno. }\end{array}$ \\
\hline $\begin{array}{l}\text { La gestión del conocimiento } \\
\text { en las organizaciones y las } \\
\text { regiones }\end{array}$ & $\begin{array}{l}\text { La gestión del conocimiento se representa como una } \\
\text { habilidad de una empresa o institución para } \\
\text { incrementar el conocimiento tácito y crear las } \\
\text { condiciones para generar el intercambio de } \\
\text { información entre los colaboradores. En la gestión } \\
\text { pública regional se han identificado la creación de las } \\
\text { ventajas competitivas. }\end{array}$ \\
\hline $\begin{array}{l}\text { Incidencia de las prácticas de } \\
\text { Gestión del conocimiento } \\
\text { sobre la creatividad } \\
\text { organizacional }\end{array}$ & $\begin{array}{l}\text { El conocimiento es un recurso fundamental, que } \\
\text { debe ser evaluado y controlado para maximizar la } \\
\text { productividad, el éxito de la gestión del } \\
\text { conocimiento está vinculado con que los nuevos } \\
\text { conocimientos mejoren las acciones de las } \\
\text { organizaciones o creen las bases para una nueva } \\
\text { acción que permitan el rendimiento creativo y } \\
\text { confluyan en un conjunto de conocimientos de la } \\
\text { empresa y generen nuevas ideas. Las prácticas de } \\
\text { aprendizaje continuo y la gestión de las } \\
\text { competencias individuales de los empleados, tienen } \\
\text { incidencia significativa sobre la creatividad, } \\
\text { considerada una fuente de ventaja competitiva y un } \\
\text { elemento indispensable para la supervivencia de las } \\
\text { organizaciones. }\end{array}$ \\
\hline $\begin{array}{l}\text { Gestión del conocimiento en } \\
\text { la administración pública de } \\
\text { los municipios de la Región } \\
\text { de la Gran Santa rosa/ RS }\end{array}$ & $\begin{array}{l}\text { En el sector público, el propósito de la gestión del } \\
\text { conocimiento no solo es mejorar el desempleo de la } \\
\text { institución pública sino más bien se extiende al } \\
\text { tratamiento de los temas relevantes consumiendo la } \\
\text { menor cantidad de recursos y tiempo. la gestión del } \\
\text { conocimiento en las organizaciones públicas de la }\end{array}$ \\
\hline
\end{tabular}




\begin{tabular}{|c|c|}
\hline & $\begin{array}{l}\text { gran región de Santa Rosa/RS, existe un gran número } \\
\text { de mujeres que asumen funciones públicas en todos } \\
\text { los niveles, ya sea como funcionarios, secretarios y } \\
\text { alcaldes, la mayor concentración de las mujeres se } \\
\text { encuentra en las áreas de salud y educación. }\end{array}$ \\
\hline $\begin{array}{l}\text { Knowledge } \text { Management In } \\
\text { Public Administration } \\
\text { Institutions }\end{array}$ & $\begin{array}{l}\text { De acuerdo con los resultados el intercambio de } \\
\text { conocimientos en las oficinas de la administración } \\
\text { pública encuestadas está principalmente influenciado } \\
\text { por la cultura organizacional, seguido de la } \\
\text { infraestructura técnica y la estructura organizativa. }\end{array}$ \\
\hline $\begin{array}{l}\text { Gestión del conocimiento en } \\
\text { el tercer sector de la } \\
\text { competitividad a la } \\
\text { eficiencia organizacional }\end{array}$ & $\begin{array}{l}\text { De acuerdo con diversos argumentos recogidos de la } \\
\text { recopilación documental, existe poca evidencia } \\
\text { empírica sobre la investigación de la gestión del } \\
\text { conocimiento en organizaciones de la gestión } \\
\text { pública, las investigaciones señalan aspectos } \\
\text { positivos para las organizaciones que emplean } \\
\text { prácticas de la gestión del conocimiento entre lo que } \\
\text { destaca la eficiencia. }\end{array}$ \\
\hline $\begin{array}{l}\text { Gestión del conocimiento y } \\
\text { uso de Tic's en la empresa } \\
\text { privada y administración } \\
\text { pública }\end{array}$ & $\begin{array}{l}\text { La gestión del conocimiento debe tener como meta el } \\
\text { uso eficiente del conocimiento para el mejoramiento } \\
\text { continuo y adecuación de sus procesos. En la mayoría } \\
\text { de las empresas se observa que una parte importante } \\
\text { de los procesos críticos de las mismas. }\end{array}$ \\
\hline
\end{tabular}

De los resultados obtenidos en la revisión bibliográfica muestran que la gestión del conocimiento es una herramienta del aprendizaje como lo manifiesta Cisneros-Cisneros et al., (2020), lo que permite a la gestión del conocimiento transferir conocimientos en salud desde las comunidades y entidades públicas, lo que le permite a las comunidades principalmente convertir la información externa como propia, es el conocimiento adquirido 
en salud asimilado que tiene influencia directa en las comunidades, en esta misma idea Echeverri et al., (2018) considera en su investigación que las prácticas de los aprendizajes continuos y la gestión de la competencias individuales de los colaboradores tienen un impacto positivo en la supervivencia de las organizaciones.

Por su parte, los autores Ceballos \& Arias, (2018), Calvo Giraldo, (2018), Bedoya-Dorado, (2015) y Ero Del Castro, et al, (2014) indican que la gestión del conocimiento permite aprovechar el conocimiento de las personas para contribuir en la organización, lo que incrementa el conocimiento tácito y genera condiciones para el intercambio de información entre colaboradores Calvo Giraldo, (2018), en esta misma idea, la investigación de BedoyaDorado, (2015) señala que las organizaciones pueden tener un impacto en la eficiencia al considerar la gestión del conocimiento, asimismo, Ero Del Castro, et al, (2014) comparte estas ideas donde evidencia que el mejoramiento continuo y adecuación de procesos puede conllevar a mejorar los procesos críticos detectados en las instituciones de la administración pública, es por ello que la gestión del conocimiento sea convertido como un buen modelo gerencial con el propósito de crear sinergias y empatía como parte de na estrategia exitosa para promover, confiabilidad, iniciativa y desarrollo personal entre los colaboradores de una institución.

Uno de los resultados indica que la gestión del conocimiento se encuentra todavía en una etapa exploratoria en el sentido de relacionarlo con el gobierno digital y la efectividad que pueda dar esta interacción, Alvarenga et al. (2020), Asimismo, para Mc Evoy et al., (2018), es difícil medir la productividad del sector público dado que se encuentra influenciado por las tendencias burocráticas, indica que el sector público presenta mayores entornos de control que el sector privado, además tiene mayor influencia externa del 
gobierno, el tesoro público y la responsabilidad social, esta mezcla compleja puede dificultar la identificación y transferencia de conocimiento en instituciones de la administración pública.

De las revisiones realizadas para Gessi et al.,(2017) la sociedad está experimentando cambios sociales y económicos que afectan a la administración pública, por ello la administración pública debe estar preparada respondiendo a través del gobierno con agilidad y calidad para el servicio del ciudadano, por ello es importante prácticas modernas como el uso de la gestión del conocimiento lo que permitirá mejorar el desempeño de la institución, asimismo, se extiende al tratamiento de los temas de importancia para la sociedad, usando un mínimo de recursos y tiempo.

Los resultados presentados en los artículos analizados evidencias claramente que la gestión del conocimiento es un elemento indispensable en la mejora de los procesos de la administración pública y que un Estado que busca mejorar debe tomar en cuenta estas modernas herramientas como la gestión del conocimiento que hacen que las experiencias de los colaboradores sean potenciadas en beneficio de la institución pública.

\section{CONCLUSIONES}

La gestión del conocimiento se ha convertido en un recurso importante de las instituciones públicas, que proveen una ventaja competitiva sostenible que contribuye al desarrollo de las instituciones de la administración pública, la gestión del conocimiento es un proceso de transformación de la información, un proceso organizado, de creación, captura, almacenamiento, diseminación y uso del conocimiento entre instituciones u organizaciones y dentro de las mismas para mantener una ventaja que les permita ser competitivos. 
En cuanto a la metodología se ha realizado una búsqueda sistematizada de literatura científica diversa lo que llevan a la conclusión de que la organización o institución en sí misma no puede crear conocimiento, lo crea los colaboradores con su talento. Para generar ese conocimiento debe ser compartido con otras personas, formando un espiral lo que representa una de las claves que hacen que la organización sea más competitiva.

La gestión del conocimiento en el sector pública engloba aspectos más amplios con el objetivo de capturar, organizar, compartir, difundir y crear conocimiento formal e informal, la implementación de la gestión del conocimiento debe ser parte de las políticas públicas, especialmente en aquellos países que desarrollan ciencia y tecnología.

De las revisiones realizadas es evidente que la gestión del conocimiento cobra especial relevancia especialmente cuando las técnicas y estrategias de las empresas son enfocadas desde el punto de vista del colaborador, lo que en todas las experiencias buscan la conversión del conocimiento en nuevo conocimiento que les permita ser entidades.

Los autores revisados coinciden que la aplicación de la gestión del conocimiento en las instituciones puede llevar a desarrollas actividades más eficientes mejorando la productividad y logrando el éxito de las instituciones.

Entre las principales fortalezas del estudio es preciso destacar que la adecuada presentación de los componentes de la gestión del conocimiento pone en evidencia la ventaja competitiva de las organizaciones o instituciones especialmente en la administración pública. 


\section{REFERENCIAS BIBLIOGRÁFICAS}

Alvarenga, A., Matos, F., Godina, R., \& Matias, J. C. O. (2020). Digital transformation and knowledge management in the public sector. Sustainability (Switzerland), 12(14). https://doi.org/10.3390/su12145824

Bedoya-Dorado, C. (2015). Gestión del conocimiento en el tercer sector: de la competitividad a la eficiencia organizacional. Entramado, 11(2), 94-111. https://doi.org/10.18041/entramado.2015v11n2.22224

Bučková, J. (2015). Knowledge Management in Public Administration Institutions. Procedia Economics and Finance, 34(15), 390-395. https://doi.org/10.1016/s2212-5671(15)01645-7

Calvo Giraldo, O. (2018). La Gestión del Conocimiento en las Organizaciones y las Regiones: Una Revisión de la Literatura. Tendencias, 19(1), 140. https://doi.org/10.22267/rtend.181901.91

Ceballos, E. J. A., \& Arias, A. V. (2018). Knowledge management, organizational policy for business today. Ingeniare, 26(4), 673-684. https://doi.org/10.4067/S0718-33052018000400673

Chávez Ramírez, E. D. (2018). Gestión del conocimiento de la plataforma web y la elección profesional de los estudiantes de una Institución Educativa, 2018. In Universidad César Vallejo.

Cisneros-Cisneros, M. A., Pemberthy-Gallo, L. S., \& Chaguendo-Muñoz, M. K. (2020). Gestión del conocimiento: una apuesta desde la gobernanza para la educación en salud en el Cauca. Opera, 27, 63-89. https://doi.org/10.18601/16578651.n27.04 
Echeverri, A., Lozada, N., \& Arias, J. E. (2018). Incidencia de las Prácticas de Gestión del Conocimiento sobre la Creatividad Organizacional. Informacion Tecnologica, 29(1), 71-82. https://doi.org/10.4067/S071807642018000100009

Ero Del Castro, Alí García, Jaime Martinez, D. U. (n.d.). 123 4. 1-16.

Gessi, N. L., Nüske, M. A., Thesing, N. J., Allebrandt, S. L., \& Baggio, D. K. (2017). Gestión del conocimiento en la administración pública de los municipios de la Región de la Gran Santa Rosa/RS. Espacios, 38(17).

Hegazy, F. M., \& Ghorab, K. E. (2014). The influence of knowledge management on organizational business processes' and employees' benefits. Vision 2020: Sustainable Growth, Economic Development, and Global Competitiveness - Proceedings of the 23rd International Business Information Management Association Conference, IBIMA 2014, 1(1), 17041733.

Mc Evoy, P. J., Ragab, M. A. F., \& Arisha, A. (2019). The effectiveness of knowledge management in the public sector. Knowledge Management Research and Practice, 17(1), 39-51. https://doi.org/10.1080/14778238.2018.1538670

Mustafa, F. H., Senoo, S., \& Pengiran Bagul, A. H. B. (2015). Knowledge management in modern aquaculture. Aquaculture Ecosystems: Adaptability and Sustainability, 281-318. https://doi.org/10.1002/9781118778531.ch9

Rubier Valdés, D. (2019). La incidencia de la gestión del conocimiento en el éxito de las organizaciones. Cooperativismo y Desarrollo, 7(3), 392-405. 
Siregar, Z. M. E., Suryana, Ahman, E., \& Senen, S. H. (2019). Does knowledge management enhance innovation: A literature review. International Journal of Scientific and Technology Research, 8(9), 19911994.

Zuluaga López, C. Mi. (2019). Capital intelectual de la Institución de Educación Superior CINOC: Contribuciones para la gestión del conocimiento. $4(3)$, $1-21$. https://media.neliti.com/media/publications/112355-ID-pengaruh-strukturaktiva-ukuranperusaha.pdf\%0Acholar.google.es/scholar?hl=es\&as_sdt=0\%2C5\&q=Funci onalidad + Familiar+en + Alumnos $+\mathrm{de}+1^{\circ}+\mathrm{y}+2^{\circ}+\operatorname{grado}+\mathrm{de}+$ secundaria $+\mathrm{de}+\mathrm{la}$ +institución+educativa+parroquial+"'Peq 